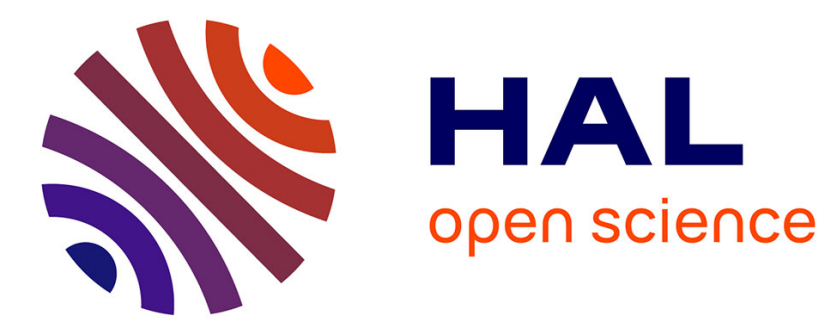

\title{
Quantum limits of optical super-resolution
}

\author{
Mikhail I. Kolobov
}

\section{To cite this version:}

Mikhail I. Kolobov. Quantum limits of optical super-resolution. Doctoral. 2008. sfo-00270561

\section{HAL Id: sfo-00270561 https://hal-sfo.ccsd.cnrs.fr/sfo-00270561}

Submitted on 6 Apr 2008

HAL is a multi-disciplinary open access archive for the deposit and dissemination of scientific research documents, whether they are published or not. The documents may come from teaching and research institutions in France or abroad, or from public or private research centers.
L'archive ouverte pluridisciplinaire HAL, est destinée au dépôt et à la diffusion de documents scientifiques de niveau recherche, publiés ou non, émanant des établissements d'enseignement et de recherche français ou étrangers, des laboratoires publics ou privés. 


\title{
ÉCOLE DE PHYSIQUE LES HOUCHES

\section{Quantum limits of optical super-resolution*}

\author{
Mikhail I. Kolobov \\ Laboratoire PhLAM, Université de Lille 1, \\ F-59655 Villeneuve d'Ascq Cedex, France \\ e-mail: mikhail.kolobov@univ-lille1.fr
}

\begin{abstract}
The conventional resolution criterion of an optical instrument was established at the end of the nineteen century in the classical works of Abbe and Rayleigh. This classical limit is based on the presumed resolution capacity of a human eye and is not a fundamental physical limit as, for example, the Heisenberg uncertainty relation. Classical superresolution techniques allow under certain conditions to go beyond the Rayleigh limit.

In this lecture we shall present the quantum theory of optical superresolution in terms of the prolate spheroidal wave functions. We shall demonstrate that the ultimate limit of super-resolution is determined by the quantum fluctuations of light in the region of the object location and the vacuum fluctuations outside it. We shall formulate the standard quantum limit of super-resolution and show that one can go beyond this limit using spatially multimode squeezed light.
\end{abstract}

*This series of lectures was delivered at Ecole Prédoctorale des Houches, session XXIV, Quantum Optics, September 10-21, 2007. The session was directed by Nicolas Treps and Isabelle Robert-Philip. 


\section{Contents}

1 Introduction $\quad 2$

2 Super-resolution in classical optics 3

2.1 Rayleigh limit, super-resolution and a priori information ... 3

2.2 Classical super-resolution . . . . . . . . . . . . . 6

3 Quantum theory of super-resolution $\quad 10$

3.1 Quantum theory of optical imaging . . . . . . . . . . . 10

3.2 Quantum limits of super-resolution . . . . . . . . . . . 13

4 Conclusions

\section{Introduction}

Today we are witnessing an extraordinary progress of digital imaging techniques for both scientific and general public applications. This rapid technological development has steered a revival of interest for resolution enhancement in imaging systems. The word "super-resolution" widely appears today is the literature in different contexts and often has different meaning. Unfortunately, this abuse of terminology has created a confusion over the concept of super-resolution.

The original idea of super-resolution has appeared in the sixties as an attempt to overcome the classical diffraction or the Rayleigh limit of resolution, imposed by diffraction in an optical system. Super-resolution is achieved when the spatial Fourier spectrum of the image can be extrapolated outside the transmission band of the imaging system. This out-of-band extrapolation can be performed when one has a priori information about the object, namely that the object is of a finite spatial extent. Indeed in such a case the spatial Fourier spectrum of the object is an entire analytical function and, therefore, it is completely determined by its part transmitted through the optical system. The out-of-band extrapolation of the spatial Fourier spectrum is equivalent of resolution beyond the Rayleigh limit. This idea of super-resolution has been widely discussed in the literature in the sixties $[1,2,3,4,5,6]$.

In the eighties, following the rapid development of digital imaging techniques, a new concept of resolution enhancement in digital imaging has 
emerged and was also called "super-resolution". However, it is very different from the original concept of super-resolution described in the previous paragraph. This new method allows to obtain a high-resolution digital image from several low-resolution digital images using a specific signal processing technique. The term "digital" reflects the fundamental difference between the two methods.

In the modern electronic imaging applications an image is detected by a charge-coupled device (CCD) or a complementary metal-oxide-semiconductor (CMOS) sensor constituted by pixels of finite size. The resolution in a detected digital image is determined not only by diffraction in the imaging system, but also by the size of the pixel in the detecting electronic device. Decreasing the size of the individual pixel one increases the resolution in the digital image. However, even with infinitely small pixels one can never improve the resolution beyond the Rayleigh limit. It is, therefore, more appropriate to call the second method of the resolution enhancement "digital deconvolution" instead of super-resolution.

Digital deconvolution technique uses multiple low-resolution digital images of the same object, shifted with respect to each other by a subpixel distance. If the low-resolution images are shifted by integer number of pixels, then each individual image contains the same information about the object and the resolution enhancement is impossible. In the case of subpixel shifts the information contained in the individual images is different, and it can be exploited to obtain a high-resolution. Recently, such a resolution enhancement approach has been one of the most active research areas in optical imaging $[7,8]$.

In this lecture we will be concerned with quantum theory of super-resolution in the sense of the resolution beyond the Rayleigh limit and not in the sense of the "digital deconvolution".

\section{Super-resolution in classical optics}

\subsection{Rayleigh limit, super-resolution and a priori infor- mation}

The classical limit of resolution of an optical instrument was formulated in the well-known works by Abbe and Rayleigh at the end of the nineteen century [9]. This classical limit states that the resolution of an optical system 
is limited by diffraction on the system pupil. Because of diffraction a point source at the input of the system creates a diffraction pattern of finite size on its output. When two point sources are placed closer and closer to each other, their diffraction patterns start to overlap and it becomes more and more difficult to discriminate these patterns. The smallest distance between two input point sources that allows for discrimination depends on many factors and is difficult to quantify. Several criteria have been proposed for such a discrimination, and the most famous one is the classical Rayleigh criterion. According to it, the diffraction patterns of two point sources are considered to be just resolved if the central maximum of the first one coincides with the first minimum of the second. For the case of the Airy pattern, corresponding to the Fraunhofer diffraction of a point source at a circular aperture, this gives the smallest distance between two input point sources equal to $R=0.61 \lambda / \alpha$, where $\lambda$ is the wavelength of the light and $\alpha$ is the ratio of the radius of the system pupil to the distance between the pupil and the image plane. The distance $R$ is known as the Rayleigh resolution limit.

As follows from this arguments, the classical Rayleigh resolution limit is based on a simple visual observation and presumed resolving capabilities of a human eye. It is not a fundamental physical limit like, for example, the speed of light or a Heisenberg uncertainty relation. Today it is recognized that modern CCD cameras allow us to achieve the performance very much exceeding that of a visual observation. For example, experimental measurements of displacements in the nanometer range has been performed to detect deflection of glass fibers [10, 11, 12], microscopic phase objects [13], movement of biological, subcellular vesicles [14], measurement of ultra-weak absorption using the mirage effect [15], or in atomic force microscopy [16]. In all these measurements the resolution is superior to the classical Rayleigh limit and is determined not by diffraction, but by different type of fluctuations in the experimental scheme. The possibility of improving the resolution beyond the diffraction limit is generally called "super-resolution" and often has different meaning. Below we shall give a rigorous definition of the term super-resolution in the sense used in this lecture.

In modern classical optics the resolution of an optical system is characterized not by the two-point Rayleigh resolution criterion, but in terms of its spatial transmission bandwidth. A typical optical system has a finite band of spatial frequencies that are transmitted through the system up to some cut-off frequency determined by the size of the system pupil. The optical system is then said to be bandlimited or diffraction-limited since diffraction 
effects on its pupil are responsible for finite resolution.

A coherent diffraction-limited imaging system in classical optics can be described by a linear equation relating the complex amplitude $a(s)$ of an input object with the complex amplitude $e(s)$ of the image [17],

$$
e(s)=\int_{-\infty}^{\infty} h\left(s, s^{\prime}\right) a\left(s^{\prime}\right) d s^{\prime}
$$

The impulse response function $h\left(s, s^{\prime}\right)$ that appears in this integral equation represents the image at point $s$ in the image plane from a point-source at point $s^{\prime}$ in the object plane. For translationally invariant or isoplanatic systems the impulse response depends only on the difference $s-s^{\prime}$ and the integral in (1) becomes convolution,

$$
e(s)=\int_{-\infty}^{\infty} h\left(s-s^{\prime}\right) a\left(s^{\prime}\right) d s^{\prime}
$$

In optics, the impulse response $h\left(s-s^{\prime}\right)$ is usually called the point-spread function (PSF) of the system, and its Fourier transform the optical transfer function (OTF). For bandlimited optical systems the OTF is identically zero outside the transmission band of the system. Super-resolution is defined as technique of restoring the spatial frequencies of the object outside the transmission band, or in other words, to enhance the resolution beyond the diffraction limit [17]. It is important to underline that in case when the object and the image fields are related by the convolution (2), super-resolution in this sense is impossible. To achieve super-resolution one needs some $a$ priori information about the input object. In this chapter we shall use as the a priori information the assumption that the object has finite spatial size. In this case, the spatial Fourier spectrum of the object is an entire analytical function and therefore, it can be completely determined by the analytic continuation from the part of the spectrum transmitted by the system pupil [1, 2]. Such out-of-band extrapolation of the spatial spectrum of the object is equivalent to the resolution enhancement beyond the Rayleigh limit.

However, such an analytic continuation of the spatial spectrum of the object is extremely sensitive to the presence of noise in the system. In fact, the problem of out-of-band extrapolation of the spatial spectrum is a typical case of the so-called "ill-posed problems" [18]. This property seriously hampers the potential of super-resolution. In practice to achieve super-resolution one has 
to detect the diffraction-limited image at the output of the optical system and then try to reconstruct the original object using specially designed numerical algorithms. In general case, an attempt to obtain significant super-resolution beyond the Rayleigh limit leads to a drastic decrease of the signal-to-noise ratio in the reconstructed object as compared to that in the original one. The main conclusions that one can derive from the numerous papers on classical super-resolution are [17]:

i) significant super-resolution in the sense of out-of-band extrapolation is possible only in the case when the size of the original object is not too large compared with the Rayleigh resolution distance;

ii) the amount of super-resolution increases logarithmically, i. e. rather weakly, with the signal-to-noise ratio in the original object.

\subsection{Classical super-resolution}

The optical scheme of diffraction-limited coherent optical imaging is shown in Fig. 1. For simplicity we consider one-dimensional case. The object of finite size $X$ is placed in the object plane. The first lens $L_{1}$ performs the spatial Fourier transform of the object into the pupil plane with a pupil of finite size $d$. Diffraction on this pupil is a physical origin of the finite resolution in our scheme (we neglect diffraction on the imaging lenses). The second lens $L_{2}$ performs the inverse Fourier transform and creates a diffraction-limited image in the image plane.

As mentioned above, to achieve super-resolution one needs some a priori information about the object. In our case we know a priori that the object is confined within the area of size $X$ and is identically zero outside. The spatial Fourier transform of such an object is an entire analytical function. Therefore, knowing the part of the Fourier spectrum within the area $d$ of the pupil allows for an analytic continuation of the total spectrum and, therefore, for unlimited resolution.

Let us introduce the dimensionless spatial coordinates in the object and the image plane as $s=2 x / X$, and in the pupil plane as $\xi=2 y / d$ (see Fig. 1). In terms of dimensionless coordinates $s$ the transformation $L$ of the classical object amplitude $a(s)$ into the classical image amplitude $e(s)$ reads:

$$
e(s)=(L a)(s)=\int_{-1}^{1} \frac{\sin \left[c\left(s-s^{\prime}\right)\right]}{\pi\left(s-s^{\prime}\right)} a\left(s^{\prime}\right) d s^{\prime}, \quad-\infty<s<\infty .
$$




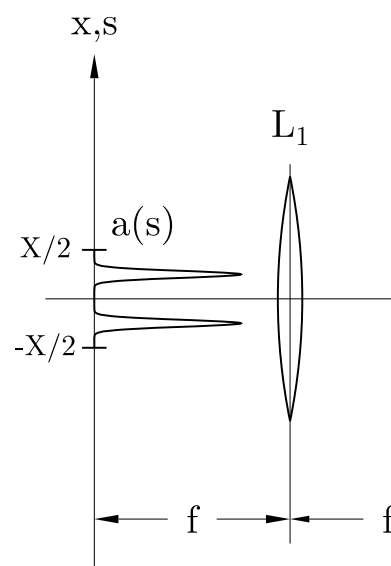

Object

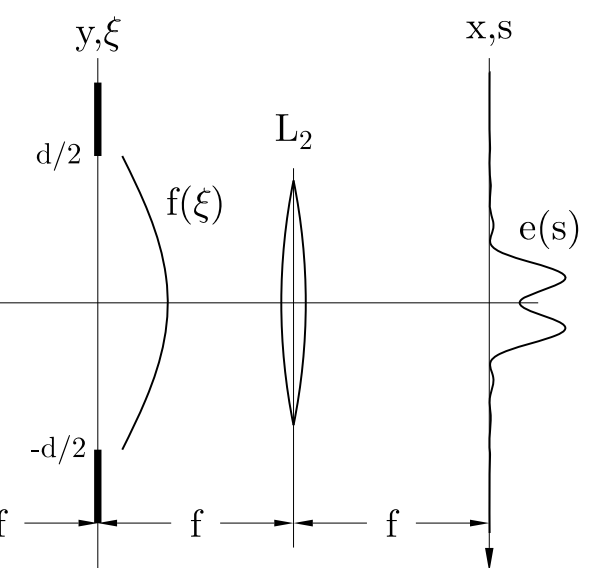

Pupil

Image

Figure 1: Optical scheme of one-dimensional coherent diffraction-limited optical imaging.

Here $c=\frac{\pi d X}{2 \lambda f}$ is the space-bandwidth product.

The problem of reconstruction of the object $a(s)$ from a detected image $e(s)$ in the absence of noise is equivalent to inversion of the integral operator $L$. The operator $L^{\star}$ adjoint to $L$ is given by [22]

$$
\left(L^{\star} f\right)(s)=\int_{-\infty}^{\infty} \frac{\sin \left[c\left(s-s^{\prime}\right)\right]}{\pi\left(s-s^{\prime}\right)} f\left(s^{\prime}\right) d s^{\prime}, \quad|s| \leq 1 .
$$

The product $A=L^{\star} L$ is the self-adjoint operator,

$$
(A f)(s)=\int_{-1}^{1} \frac{\sin \left[c\left(s-s^{\prime}\right)\right]}{\pi\left(s-s^{\prime}\right)} f\left(s^{\prime}\right) d s^{\prime}, \quad|s| \leq 1,
$$

studied by Slepian and Pollak [19]. The orthonormal system of eigenfunctions of $A$ is given by

$$
\varphi_{k}(s)=\left\{\begin{array}{cl}
\frac{1}{\sqrt{\lambda_{k}}} \psi_{k}(s) & |s| \leq 1 \\
0 & |s|>1
\end{array}\right.
$$


where $\psi_{k}(s)$ are the linear prolate spheroidal functions $[19,20]$, and $\lambda_{k}$ are the corresponding eigenvalues. The functions $\varphi_{k}(s)$ form a basis in $L^{2}(-1,1)$ and may be considered as "elements of information" of the input object. The eigenvalues $\lambda_{k}$ are an infinite set of real, positive numbers obeying $1 \geq \lambda_{0}>$ $\lambda_{1}>\ldots>0$. For small $k$ the $\lambda_{k}$ fall off slowly with $k$ until the index reaches the critical value, $k=S$, called the Shannon number,

$$
S=\frac{2 c}{\pi}=\frac{d X}{\lambda f}
$$

beyond which the $\lambda_{k}$ rapidly approach zero. In Fig. 2 we show the first 17 prolate functions $\varphi_{k}(s)$ together with corresponding eigenvalues $\lambda_{k}$ for $c=1$.

Using the properties of prolate spheroidal functions,

$$
\begin{gathered}
\int_{-1}^{1} \frac{\sin \left[c\left(s-s^{\prime}\right)\right]}{\pi\left(s-s^{\prime}\right)} \psi_{k}\left(s^{\prime}\right) d s^{\prime}=\lambda_{k} \psi_{k}(s), \\
\int_{-\infty}^{\infty} \frac{\sin \left[c\left(s-s^{\prime}\right)\right]}{\pi\left(s-s^{\prime}\right)} \psi_{k}\left(s^{\prime}\right) d s^{\prime}=\psi_{k}(s),
\end{gathered}
$$

we obtain

$$
L \varphi_{k}=\sqrt{\lambda_{k}} \psi_{k}, \quad L^{\star} \psi_{k}=\sqrt{\lambda_{k}} \varphi_{k} .
$$

Expanding the object amplitude over the functions $\varphi_{k}(s)$ and the image amplitude over $\psi_{k}(s)$, we can easily find the relation between the expansion coefficients of the object and the image. Indeed, since the functions $\varphi_{k}(s)$ form a complete orthonormal set in $[-1,1]$ we can write the object amplitude as

$$
a(s)=\sum_{k=0}^{\infty} a_{k} \varphi_{k}(s), \quad|s| \leq 1,
$$

with the coefficients $a_{k}$ given by

$$
a_{k}=\int_{-\infty}^{\infty} a(s) \varphi_{k}(s) d s
$$

Similar expansion can be written for the image amplitude in terms of functions $\psi_{k}(s)$

$$
e(s)=\sum_{k=0}^{\infty} e_{k} \psi_{k}(s), \quad-\infty<s<\infty
$$



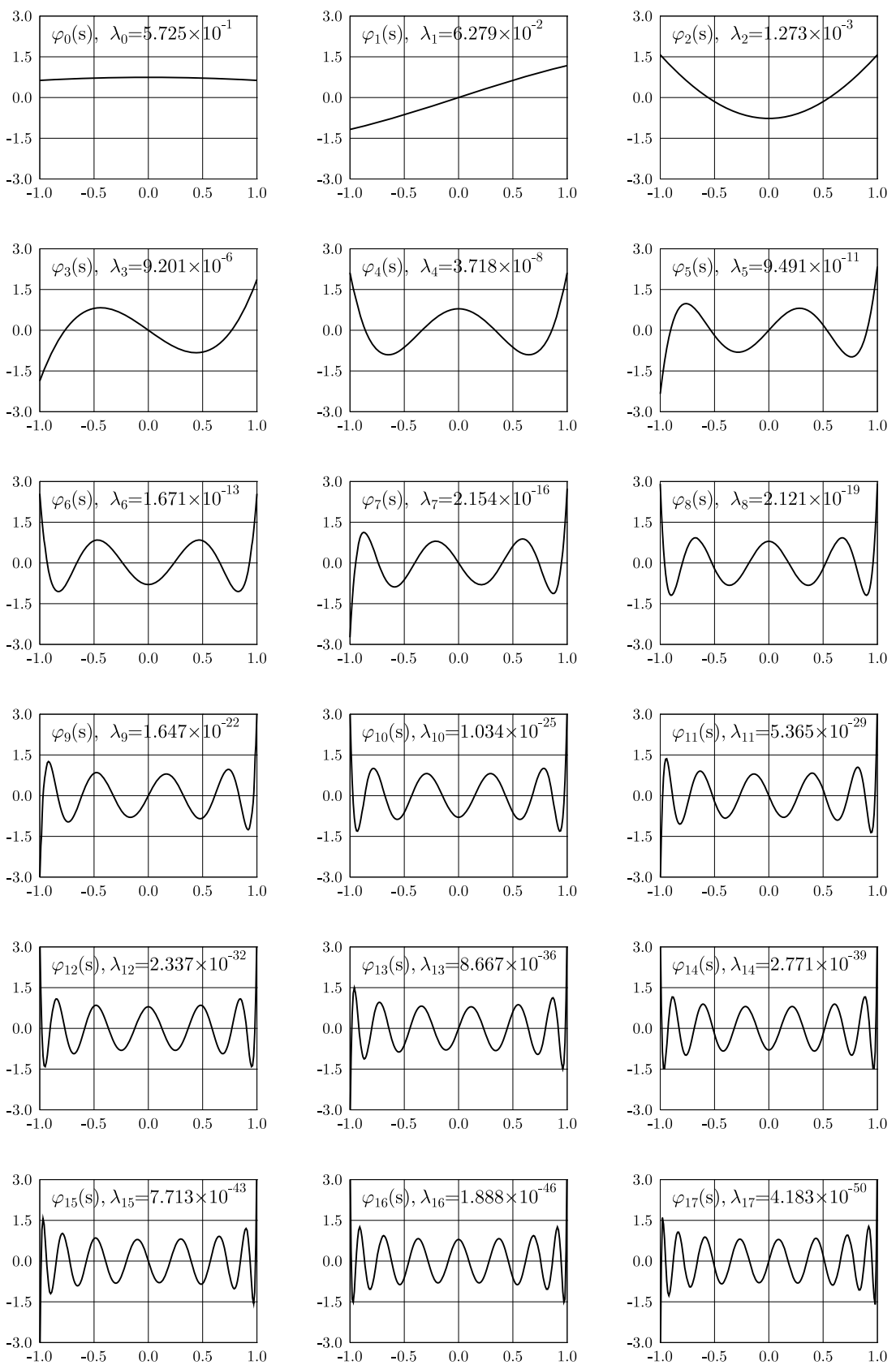

Figure 2: Examples of the prolate spheroidal functions $\varphi_{k}(s)$ and the corresponding eigenvalues $\lambda_{k}$. 
with the coefficients $e_{k}$ given by

$$
e_{k}=\int_{-\infty}^{\infty} e(s) \psi_{k}(s) d s
$$

Substituting these expansions into Eq. (3) and using the first of Eqs. (10) we obtain the following relation between $a_{k}$ and $e_{k}$ :

$$
e_{k}=\sqrt{\lambda_{k}} a_{k}
$$

Let us assume that we can detect the classical field profile $e(s)$ in the image plane. Then using Eqs. (14), (15)we can reconstruct the coefficients $a_{k}^{(r)}$, where $(r)$ stands for "reconstructed", as

$$
a_{k}^{(r)}=\frac{e_{k}}{\sqrt{\lambda_{k}}}=a_{k}
$$

This equation demonstrates that, if we neglect the fluctuations in the imaging and detection scheme, the reconstruction coefficients $a_{k}^{(r)}$ are identical with the coefficients of the input image $a_{k}$. This is equivalent to unlimited superresolution. In order to establish the quantum limits of super-resolution we have to determine the role of quantum fluctuations of light in the imaging and the detection process.

\section{Quantum theory of super-resolution}

\subsection{Quantum theory of optical imaging}

In the quantum theory the classical object amplitude $a(s)$ becomes the dimensionless photon annihilation operator in the object plane $\hat{a}(s)$ and the classical image amplitude $e(s)$ the corresponding photon annihilation operator in the image plane $\hat{e}(s)$. These operators obey the standard commutation relations,

$$
\left[\hat{a}(s), \hat{a}^{\dagger}\left(s^{\prime}\right)\right]=\delta\left(s-s^{\prime}\right), \quad\left[\hat{e}(s), \hat{e}^{\dagger}(s)\right]=\delta\left(s-s^{\prime}\right),
$$

and are normalized so that $\left\langle\hat{a}^{\dagger}(s) \hat{a}(s)\right\rangle$ gives the mean photon number per unit dimensionless length in the object plane and $\left\langle\hat{e}^{\dagger}(s) \hat{e}(s)\right\rangle$ - in the image plane. 
In the quantum theory we can use Eqs. (11),(13) now treating the expansion coefficients $a_{k}$ and $e_{k}$ as photon annihilation operators $\hat{a}_{k}$ and $\hat{e}_{k}$. Using the properties of the prolate spheroidal functions it can be shown that the operators $\hat{a}_{k}$ in the object plane obey the following commutation relations:

$$
\left[\hat{a}_{k}, \hat{a}_{l}^{\dagger}\right]=\delta_{k l}, \quad\left[\hat{a}_{k}, \hat{a}_{l}\right]=0 .
$$

The same commutation relations must be satisfied by the photon annihilation operators $\hat{e}_{k}$ in the image plane. However, Eq. (15) does not preserve the commutation relations (18). The reason for this is that classical imaging equation (3) takes into account only nonzero field amplitude in the region $|s| \leq 1$ of the object plane. The rest of this plane $|s|>1$ is ignored because there the classical field amplitude is zero. In the quantum theory this region must be taken into account to guarantee the conservation of the commutation relations.

To obtain the canonical transformation of the photon annihilation and creation operators from the object into the image plane we shall split the coordinate $s$ into two regions, the "core", $|s| \leq 1$, corresponding to the area of localization of the classical object, and the "wings", $|s|>1$, outside these area. The orthonormal bases in these areas of the object plane are given by [21]

$$
\varphi_{k}(s)=\left\{\begin{array}{cl}
\frac{1}{\sqrt{\lambda_{k}}} \psi_{k}(s) & |s| \leq 1, \\
0 & |s|>1,
\end{array} \quad \chi_{k}(s)=\left\{\begin{array}{cl}
0 & |s| \leq 1 \\
\frac{1}{\sqrt{1-\lambda_{k}}} \psi_{k}(s) & |s|>1 .
\end{array}\right.\right.
$$

In terms of two sets $\left\{\varphi_{k}(s)\right\}$ and $\left\{\chi_{k}(s)\right\}$ we can write the annihilation operators in the object plane as

$$
\hat{a}(s)=\sum_{k=0}^{\infty} \hat{a}_{k} \varphi_{k}(s)+\sum_{k=0}^{\infty} \hat{b}_{k} \chi_{k}(s) .
$$

Here $\hat{b}_{k}$ are the annihilation operators of the prolate modes $\chi_{k}$ in the wings region, expressed through the field operator $\hat{a}(s)$ by

$$
\hat{b}_{k}=\int_{-\infty}^{\infty} \hat{a}(s) \chi_{k}(s) d s
$$

The photon annihilation operator $\hat{e}(s)$ in the image plane can be written in 
terms of functions $\psi_{k}(s)$

$$
\hat{e}(s)=\sum_{k=0}^{\infty} \hat{e}_{k} \psi_{k}(s)+\hat{e}_{\perp}(s), \quad-\infty<s<\infty,
$$

with the coefficients $\hat{e}_{k}$ given by

$$
\hat{e}_{k}=\int_{-\infty}^{\infty} \hat{e}(s) \psi_{k}(s) d s .
$$

In Eq. (22) we have added the term $\hat{e}_{\perp}(s)$ in order to satisfy the commutation relations (17). However, this term is orthogonal to the expansion over the prolate spheroidal functions $\psi_{k}(s)$ and, therefore, will not appear in the transformation below.

Substituting the expansions (22), (20) into Eq. (3) we obtain the following relation between the photon annihilation operators in the object and the image plane,

$$
\hat{e}_{k}=\sqrt{\lambda_{k}} \hat{a}_{k}+\sqrt{1-\lambda_{k}} \hat{b}_{k}
$$

It is easy to verify that this transformation preserves the commutation relations of the operators, $\left[\hat{a}_{k}, \hat{a}_{l}^{\dagger}\right]=\left[\hat{b}_{k}, \hat{b}_{l}^{\dagger}\right]=\left[\hat{e}_{k}, \hat{e}_{l}^{\dagger}\right]=\delta_{k l}$.

Equation (24) is completely equivalent to the transformation performed by a beam splitter. Indeed, if we consider the operators $\hat{a}_{k}$ and $\hat{b}_{k}$ as the photon annihilation operators in the modes defined by prolate spheroidal waves incoming to the beam splitter with the amplitude transmission coefficient $\sqrt{\lambda_{k}}$ and the reflection coefficient $\sqrt{1-\lambda_{k}}$, then $e_{k}$ is the photon annihilation operator in the $k$ th mode of the transmitted wave.

$>$ From Fig. 1 one may think that the vacuum fluctuations coming into the image plane from the region $|\xi|>1$ of the Fourier plane outside the pupil should be also taken into account. Indeed, when treating the field in the Fourier plane as an operator we must include the contribution from this region into the resulting field in the image plane. However, the advantage of expansion (13) is that the field from this region does not contribute to the expansion coefficients $\hat{e}_{k}$ of the image because it is orthogonal to the prolate spheroidal wave functions. This property was pointed out by Bertero and Pike in [22] for the out-of-band classical noise and remains valid in the quantum theory.

Let us assume that we can detect the photon annihilation operators $\hat{e}(s)$ in the image plane using a sensitive CCD camera. It should be emphasized 
that, since we need the complex field amplitudes and not the intensities, one should use the homodyne detection scheme with a local oscillator. Then we can calculate the operator-valued coefficients $\hat{a}_{k}^{(r)}$ of the reconstructed object as

$$
\hat{a}_{k}^{(r)}=\frac{\hat{e}_{k}}{\sqrt{\lambda_{k}}}=\hat{a}_{k}+\sqrt{\frac{1-\lambda_{k}}{\lambda_{k}}} \hat{b}_{k} .
$$

As follows from Eq. (25), the reconstruction of the input object is not exact because of the second term in Eq. (25). This term contains the annihilation operators $\hat{b}_{k}$ responsible for the vacuum fluctuations of the electromagnetic field in the area outside the object. It is important to notice that these vacuum fluctuations prevent from reconstruction of the higher and higher coefficients $\hat{a}_{k}$ in the object because of the multiplicative factor $\sqrt{\left(1-\lambda_{k}\right) / \lambda_{k}}$. Indeed, the eigenvalues $\lambda_{k}$ become rapidly very small after the index $k$ has attained some critical value. This leads to rapid "amplification" of the vacuum fluctuations in the reconstructed object that limits the number of the reconstructed coefficients $\hat{a}_{k}$.

\subsection{Quantum limits of super-resolution}

To draw the general conclusions applicable to different kinds of squeezed light used as a coherent source for imaging, we shall assume that detection of the image in the image plane is organized in such a way that it allows to detect one of two quadrature components of the image field. In quantum optics this is achieved by using the homodyne detection technique mixing the image field in the detection plane with plane coherent local oscillator field. Therefore, we shall decompose the field operator $\hat{e}_{k}$ into its quadrature components

$$
\hat{e}_{k}=\hat{e}_{1 k}+i \hat{e}_{2 k}
$$

and will investigate their fluctuations.

As follows from Eq. (24), the fluctuations of the image quadrature components are related to those of the object and the vacuum fluctuations outside the object,

$$
\left\langle\left(\Delta \hat{e}_{\mu k}\right)^{2}\right\rangle=\lambda_{k}\left\langle\left(\Delta \hat{a}_{\mu k}\right)^{2}\right\rangle+\left(1-\lambda_{k}\right)\left\langle\left(\Delta \hat{b}_{\mu k}\right)^{2}\right\rangle,
$$

with $\mu=1,2$ for corresponding quadratures. In turn, the fluctuations in the quadrature components of the reconstructed image can be calculated using 
Eq. (25) as follows

$$
\left\langle\left(\Delta \hat{a}_{\mu k}^{(r)}\right)^{2}\right\rangle=\frac{\left\langle\left(\Delta \hat{e}_{\mu k}\right)^{2}\right\rangle}{\lambda_{k}} .
$$

Let us calculate these fluctuations for three different cases:

1) the object in a coherent state and the vacuum outside the "physical object":

$$
\left\langle\left(\Delta \hat{a}_{\mu k}\right)^{2}\right\rangle=\left\langle\left(\Delta \hat{b}_{\mu k}\right)^{2}\right\rangle=\frac{1}{4} .
$$

We obtain the following result,

$$
\left\langle\left(\Delta \hat{a}_{\mu k}^{(r)}\right)^{2}\right\rangle=\frac{1}{4 \lambda_{k}}
$$

which corresponds to the standard quantum limit of super-resolution.

2) the object in a coherent state and the squeezed vacuum outside the object:

$$
\left\langle\left(\Delta \hat{a}_{\mu k}\right)^{2}\right\rangle=\frac{1}{4}, \quad\left\langle\left(\Delta \hat{b}_{\mu k}\right)^{2}\right\rangle=\frac{1}{4} e^{ \pm 2 r} .
$$

In this case we obtain,

$$
\left\langle\left(\Delta \hat{a}_{\mu k}^{(r)}\right)^{2}\right\rangle=\frac{1}{4}+\frac{1-\lambda_{k}}{\lambda_{k}} \frac{1}{4} e^{ \pm 2 r},
$$

an improvement beyond the standard quantum limit for the properly chosen quadrature component.

3) the object in a squeezed state and the squeezed vacuum outside the object:

$$
\left\langle\left(\Delta \hat{a}_{\mu k}\right)^{2}\right\rangle=\left\langle\left(\Delta \hat{b}_{\mu k}\right)^{2}\right\rangle=\frac{1}{4} e^{ \pm 2 r}
$$

This provides,

$$
\left\langle\left(\Delta \hat{a}_{\mu k}^{(r)}\right)^{2}\right\rangle=\frac{1}{4 \lambda_{k}} e^{ \pm 2 r},
$$

further resolution improvement beyond the standard quantum limit with multimode squeezed light. 


\section{Conclusions}

Classical concept of super-resolution allows to improve optical resolution of an imaging system beyond the Rayleigh limit using a priori information about the finite spatial extent of the object. The amount of super-resolution depends on two physical parameters of the imaging and detection system: the signal-to-noise ratio, and the space-bandwidth product.

We have formulated the quantum theory of optical super-resolution that allows to establish the ultimate performance limit of this method, imposed by the quantum nature of light. In particular, we have obtained the standard quantum limit of super-resolution and have shown that one can go beyond this limit using multimode squeezed states of light. 


\section{References}

[1] H. Wolter, in Progress in Optics Vol. I, edited by E. Wolf (NorthHolland, Amsterdam, 1996), ch. V.

[2] J. L. Harris, J. Opt. Soc. Am. 54, 931 (1964).

[3] G. Toraldo di Francia, J. Opt. Soc. Am. 45, 497 (1955).

[4] C. W. McCutchen, J. Opt. Soc. Am. 57, 1190 (1967).

[5] C. K. Rushforth and R. W. Harris, J. Opt. Soc. Am. 58, 539 (1968).

[6] G. Toraldo di Francia, J. Opt. Soc. Am. 59, 799 (1969).

[7] S. Farsiu, D. Robinson, M. Elad, and P. Milanfar, Int. J. Imaging Syst. Technol. 14, 47 (2004).

[8] S. C. Park, M. K. Park and M. G. Kang, IEEE Signal Process. Mag. 20, 21 (2003).

[9] J. Rayleigh, in Collected Optics Papers of Lord Rayleigh, part A, (Opt. Soc. Am., Washington,1994), p. 117.

[10] A. Flock and D. Strelioff, Nature, 310, 397 (1984).

[11] J. J. Art, A. C. Craftford, and R. Fettiplace, J. Physiol. (London)371, 18P (1986).

[12] S. Kamimura, Appl. Opt. 26, 3425 (1987).

[13] W. Denk and W. W. Webb, Appl. Opt. 29, 2382 (1990).

[14] J. Jelles, B. J. Schnapp, and M. P. Scheetz, Nature, 331, 450 (1988).

[15] D. Fournier, A. Boccara, N. Amer, and R. Gerlach, Appl. Phys. Lett. 37, 519 (1980).

[16] C. Putman, B. De Grooth, N. Van Hulst, and J. Greve, J. Appl. Phys. 72, 6 (1992).

[17] M. Bertero, and C. De Mol, in Progress in Optics Vol. XXXVI, edited by E. Wolf (North-Holland, Amsterdam, 1996), p. 129. 
[18] A. N. Tikhonov and V. Y. Arsenin, Solution of ill-posed problems (Winston/Wiley, Washington, DC, 1977).

[19] D. Slepian and H. O. Pollak, Bell System Techn. J. 40, 43 (1961).

[20] B. R. Frieden, in Progress in Optics, Vol. IX, E. Wolf, ed. (NorthHolland, Amsterdam, 1971), pp. 311-407.

[21] M. I. Kolobov and C. Fabre, Phys. Rev. Lett. 85, 3789 (2000).

[22] M. Bertero and E. R. Pike, Opt. Acta 29, 727 (1982). 